\title{
Error in statistical tests of error in statistical tests Monwhea Jeng*1,2
}

Address: ${ }^{1}$ Department of Physics, Box 1654, Southern Illinois University Edwardsville, Edwardsville, IL 62025 and ${ }^{2}$ Department of Physics, Syracuse University, Syracuse, NY 13244

Email: Monwhea Jeng* - mjeng@physics.syr.edu

* Corresponding author

Published: 13 September 2006

BMC Medical Research Methodology 2006, 6:45 doi:10.1186/147I-2288-6-45

This article is available from: http://www.biomedcentral.com/I47/-2288/6/45

(c) 2006 Jeng; licensee BioMed Central Ltd.

This is an Open Access article distributed under the terms of the Creative Commons Attribution License (http://creativecommons.org/licenses/by/2.0), which permits unrestricted use, distribution, and reproduction in any medium, provided the original work is properly cited.
Received: 25 January 2006

Accepted: 13 September 2006

\begin{abstract}
Background: A recent paper found that terminal digits of statistical values in Nature deviated significantly from an equiprobable distribution, indicating errors or inconsistencies in rounding. This finding, as well as the discovery that a large percentage of $p$ values were inconsistent with reported test statistics, led to a great deal of concern in the popular press and scientific community. The findings ultimately led to new guidelines for all Nature Research Journals.
\end{abstract}

Methods: We checked the statistical analysis behind the original paper's tests of equiprobability.

Results: The original paper tested equiprobability with the Kolmogorov-Smirnov test outside its regime of validity. Correct tests find no statistically significant deviations from equiprobability for the statistical values in Nature.

Conclusion: Statistical tests should be used correctly.

\section{Background}

A recent paper concluded that "statistical practice is generally poor, even in the most renowned scientific journals" [1]. The paper prompted significant attention in the popular press, and serious concern within the scientific community [2-7]. It led the editors of Nature Medicine to review their statistical practices, ultimately resulting in new statistical guidelines for all Nature Research Journals [3].

One of the two main results of [1] was that terminal digits of statistical values in Nature deviated significantly from an equiprobable distribution, indicating errors or inconsistencies in rounding. The authors of [1] collected random samples of test statistics and $p$ values published in Nature, and looked at the terminal digits of these numbers. Their raw data is shown in tables 1 and 2. They argued that these terminal digits should be spread evenly among the ten possible digits. Applying the KolmogorovSmirnov test with SPSS for Windows, they obtained $Z=$ $2.7, p<0.0005$, for the 610 test statistics, and $Z=1.4, p=$

Table I: Terminal digits of test statistics from volumes 409-4I2 of Nature

\begin{tabular}{ll}
\hline Digit & Frequency \\
\hline 0 & 67 \\
1 & 67 \\
2 & 65 \\
3 & 71 \\
4 & 51 \\
5 & 58 \\
6 & 53 \\
7 & 61 \\
8 & 62 \\
9 & 55 \\
\hline
\end{tabular}


Table 2: Terminal digits of $p$ values from volumes $409-412$ of Nature

\begin{tabular}{ll}
\hline Digit & Frequency \\
\hline 0 & 10 \\
1 & 20 \\
2 & 25 \\
3 & 24 \\
4 & 12 \\
5 & 16 \\
6 & 25 \\
7 & 20 \\
8 & 16 \\
9 & 13
\end{tabular}

0.043 , for the $181 p$ values. They thus concluded that the terminal digits suffered from errors, most likely due to poor rounding procedures. We point out that the original paper's test of equiprobability was based on invalid use of the Kolmogorov-Smirnov test on categorical data and that correct statistical testing finds no statistically significant deviations from equiprobability.

The authors of [1] also found a number of cases where $p$ values in Nature and the British Medical Journal were reported incorrectly, based on comparison with the reported test statistics. That finding is unaffected by our analysis.

\section{Methods}

We ran tests of equiprobability on terminal digits of the test statistics and $p$ values in Nature, using both $\chi^{2}$ tests, and a modification of the Kolmogorov-Smirnov test for categorical data.

\section{Results and Discussion}

The Kolmogorov-Smirnov test is normally used to test whether data follows a specified continuous distribution [8]. A simple calculation from the raw data in [1] shows that the $Z$ and $p$ values obtained there are those based on comparing the data with a distribution uniform on the continuous interval $[0,9]$. But this distribution is obviously incorrect even before any comparison with the data, since the terminal digit cannot be, for example, 2.68. A check of the documentation for SPSS for Windows confirms that the program runs the Komogorov-Smirnov test for a continuous uniform distribution, rather than a discrete uniform distribution.

Because the terminal digits are naturally discrete, a $\chi^{2}$ test is appropriate [8]. $\chi^{2}$ tests yield $\chi^{2}=6.5, \mathrm{df}=9, p=0.69$, for the 610 test statistics, and $\chi^{2}=15, \mathrm{df}=9, p=0.086$, for the $181 p$ values. This changes the results from "significant" to "not significant," and we therefore have insuffi- cient evidence to suggest terminal digit errors in the $p$ values reported in Nature articles.

Because the reader may be suspicious that this is simply a judgment call as to the most natural statistical test, rather than a bona fide mistake in [1], we also rerun the Kolmogorov-Smirnov test. While it is unusual to run this test on discrete data, it is possible (although perhaps poorly motivated in this case), so long as appropriate modifications are made. Instead of incorrectly comparing the data against the distribution $P(x)=1 / 9$ for $0 \leq x \leq 9$, we use $P(x)$ $=(1 / 10) \sum_{j=0}^{9} \delta(x-j)$. This gives $Z=1.1$ for the 610 test statistics $(\Delta=0.043)$, and $Z=0.60$ for the $181 p$ values $(\Delta$ $=0.045)$. Since the textbook tables of Kolmogorov-Smirnov $p$ values are computed for continuous distributions, we convert from $Z$ to $p$ values with Monte Carlo simulations (counting ties in $Z$ as "half a hit"). This gives $p=$ 0.094 and $p=0.57$ for the two cases. Again, the terminal digit distributions could reasonably have occurred by chance, given a discrete uniform distribution.

The authors of [1] also found that 21 of the $181 p$ values in Nature had problems when compared with the corresponding test statistics. Our analysis does not change this finding, but it is worth remarking on the comparatively minor nature of many of the problems that they found. For example, 3 of the 21 problems come from a single three-row table (table 1 of [9]), in which every entry of a column labelled $P$ reads 0.001 . This is indeed somewhat misleading, since the natural implication is that $p=0.001$ for all three entries, when in fact the intended meaning of the table was (presumably) that all three results were significant at the $0.1 \%$ level (i.e. $p<0.001$ ). But it is hard to imagine that many readers would be badly misled by this table, and in any event, such errors are minor in comparison to the error of using a demonstrably invalid test.

\section{Conclusion}

The authors of [1] concluded that statistical tests in papers need to be inspected more closely. However, one of the main findings of their paper is invalidated by incorrect use of a statistical test. It is ironic that despite the great attention that their paper has attracted over the last two years, this error has escaped notice. While their paper still points to the need for greater scrutiny of statistics, that scrutiny would be better directed at the assumptions used in the statistical tests, rather than at the precise $p$ values obtained.

\section{Competing interests}

The author(s) declare that they have no competing interests. 


\section{Authors' contributions}

$\mathrm{MJ}$ is responsible for this work in its entirety.

\section{Acknowledgements}

None to declare.

\section{References}

I. García-Berthou E, Alcaraz C: Incongruence between test statistics and $\mathbf{P}$ values in medical papers. BMC Medical Research Methodology 2004, 4:13.

2. Pearson $\mathrm{H}$ : Double check casts doubt on statistics in published papers. Nature 2004, 429:490.

3. Editorial: Statistically Significant. Nature Medicine 2005, I I:I.

4. Abbasi K: Editor's choice: Do mistakes matter? BMJ 2004, 328:0.

5. Sloppy stats shame science. The Economist :74. 5 Jun 2004

6. Coghlan A: Statistical flaws revealed in top journals' papers. NewScientist.com news service . 28 May 2004

7. Matthews R: Errors behind fluke results. Financial Times. 9 Jul 2004

8. Press WH, Teukolsky SA, Vetterling WT, Flannery BP: Numerical Recipes in C 2nd edition. USA: Cambridge University Press; 1992:620-628.

9. Kiesecker JM, Blaustein AR, Belden LK: Complex causes of amphibian population declines. Nature 200I, 4 I 0:68I-684.

\section{Pre-publication history}

The pre-publication history for this paper can be accessed here:

http://www.biomedcentral.com/1471-2288/6/45/prepub

Publish with Biomed Central and every scientist can read your work free of charge

"BioMed Central will be the most significant development for disseminating the results of biomedical research in our lifetime. "

Sir Paul Nurse, Cancer Research UK

Your research papers will be:

- available free of charge to the entire biomedical community

- peer reviewed and published immediately upon acceptance

- cited in PubMed and archived on PubMed Central

- yours - you keep the copyright

Submit your manuscript here:

http://www.biomedcentral.com/info/publishing_adv.asp 